Aus der chirurgisehen Abtheilung der Magdeburger Krankenanstalt Sudenburg. (Dirigirender Arzt: Dr. Rudolf Habs.)

\title{
Ein Fall von isolirter Durchschneidung des Nervus sympathicus bei Stichverletzung.
}

\author{
Von \\ Dr. Georg van der Briele, \\ Assistenzarzt.
}

Am 2. December 1901. wurde der Schlosser A. J., 19 Jahre alt, in die Magdeburger Krankenanstalt Sudenburg aufgenommen. Derselbe machte folgende Angaben. Am 1. December 1901 Abends gegen $10 \mathrm{Uhr}$ hätten einige junge Leate auf der Strasse mit ihm Händel angefangen. Plötzlich habe er das Gefuhl gehabt, als ob er einen Schlag ins Gesicht bekommen hätte, darauf habe er einen starken Blutverlust aus einer Wunde am Halse gehabt und sei ohnmächtig geworden. Er erhielt einen Nothverband und liess sich auf Anrathen seines Kassenarztes am folgenden Tage in die Anstalt aufnehmen.

Status praesens vom 2. December 1901 .

Mittelgrosser kräftiger Mann von gutem Ernährungszustand. Innere Organe ohne wesentliche Veränderungen. Facialisinnervation intact.

Anf der rechten Wange zwischen dem vorderen Rande des Musculus sternocleidomastoideus und dem vorderen Rande des äusseren Ohres befindet sich eine linienförmige horizontale $1 \mathrm{~cm}$ lange Wunde mit glatten Rändern, welche anch den untersten Theil des Obrläppcheus durchtrennt hat. Zur Sicherstellung der Diagnose wird eine Sonde in die Wunde eingefuhrt. Dieselbe geht in horizontaler und frontaler Richtung mit geringer Abweichung nach hinten und unten mehrere Centimeter weit in die Tiefe. Die Spitze gelangt bis zur Vorderfäche der Halswirbelsäule, wo man sie mit dem Finger vom Munde aus durchfühlen kann. Bei der Besichtigung fällt auf, dass die rechte Pupille bedeutend enger ist als die linke, was nach Angabe des Patienten und seiner Angehörigen früher nicht der Fall gewesen sein soll. Beide Pupillen reagiren prompt auf Licht und Accommodation. Das rechte Augenlid hängt tiefer als das linke. Die Injection der Augenbindehäute ist beiderseits gleich stark. Ebenso ist in der Spannung der beiden Augäpfel ein Unterschied nicht zu constatiren. Ein Unterschied in der Färbung (Gefässinjection) der beiden Gesichtshälften besteht nicht, auch ist eine Verschiedenheit der Hauttemperatur beider Seiten nicht wahrzunehmen. Kein vermehrter Speichelfluss. Puls und Athmung zeigen durchaus normales Verhalten. Patient klagt über Beschwerden beim Schlucken. Beim Geniessen fester und flüssiger Speisen verschluckt er sich leicht. Die hintere Rachenwand ist stark geröthet, auf derselben mehrere kleinere Suggillationen. 
Die Uvula weicht nach rechts ab. Am 11. December 1901 wurde der Patient Herrn Dr. Paul Schreiber, Augenarzt in Magdeburg, vorgefuhrt, welcher folgendes feststellte: Myosis auf der rechten Seite, die Pupillen verhalten sich wie $3: 4$. Die Pupille des rechten Auges reagirt nicht anf Hautreize, wohl aber auf Licht und Accommodation. Herabsetzung des intraoculären Druckes besteht nicht. Das rechte Auge prominirt vor dem linken um $2 \mathrm{~mm} .{ }^{1}$ ) Leichte Ptosis ist rechts deutlich. Die Lidspaltenweite beträgt links $10 \mathrm{~mm}$, rechts $8 \mathrm{~mm}$. Röthung der betreffenden Gesichtshälfte, sowie pathologische Transpiration nicht zu constatiren. Sehschärfe und ophthalmologischer Befund beiderseits normal. 14. December 1901. Die Wunde ist per primam intentionem geheilt. Schluckbeschwerden bestehen nicht mehr. Myosis Ptosis, Exophthalmus bestehen noch in demselben Grade wie bei der Aufnahme.

Patient wird auf Wunsch fast geheilt entlassen.

Auf Grund des angegebenen Befundes müssen wir die Diagnose auf Durchschneidung des Halstheils des Nerrus sympathicus oberhalb des Ganglion supremum stellen.

Zunächst entspricht der Verlauf des Stichkanals genau der topographisch-anatomischen Lage des Nerven. Der Kannal beginnt am vorderen Rande des Musculus sternocleidomostoideus genau an der Stelle, wo man auch in den meisten Fällen bei der Resection den Hautschnitt macht, und führt nach der Vorderfläche der Halswirbelsäule. Weiterhin können wir die Diagnose auch aus den Lehren der Physiologie begründen. Der Halstheil des Sympathicus hat viererlei Functionen. Er führt pupillenerweiternde Fasern, Bewegungsfasern für die glatte Musculatur der Augenhöhlen, vasomotorische Fasern für das Gesieht, die Conjunctiva, die Retina, sowie schliesslich secretorische Fasern für die Speicheldrüsen, und die Schweissdrüsen des Gesichts. Es muss also die Durchschneidung des Sympathicus eine Lähmung der Pupillenerweiterer bewirken und somit eine Verengerung der Pupillen der verletzten Seite herbeiführen. In unserem Falle haben wir dieses Symptom sehr deutlich ausgesprochen. Wir haben hier einen messbaren Unterschied der Sehlöcher auf der gesunden und verletzten Seite. Ferner führt der Nerv Bewegungsfassern für die glatten Muskeln der Augenhöhle. Man sollte also erwarten, dass nach der Durchtrennung ein Enophthalmus auftreten müsste, weil der Tonus der betreffenden Muskeln aufgehoben werden müsste. Wir haben dagegen in unserem Falle keinen Enophthalmus, sondern im Gegentheil einen Exophthalmus. B $\mathrm{rau} \mathrm{n}$ in Göttingen, welcher in 9 Fällen den Sympathicus theils einseitig, theils doppelseitig resecirt hat, um dadurch eine Heilung der genuinen Epilepsie herbeizuführen, und welcher die dabei auftretenden Er-

1) Gemessen mit dem Dr. Schreiber'sehen Exophthalmometer.

Deatsche Zeitschrift t. Chirargie. Bd. LXIV. 
98 V. van Der Brteis, Ein Fall von isolirter Durchschneidung des Nervus symp.

scheinungen sehr genau studirt hat, hat niemals Enophthalmus beobachten können. Dasselbe gilt von dem Unveränderlichbleiben der Spannung des Bulbus.

Ferner haben wir noch eine Ptosis des oberen Lides auf Seiten der Verletzung beobachtet, welche durch Lähmung der glatten Muskelfasern des Musculus palpebrae superioris bedingt ist. Vasomotorische Störungen haben wir nicht beobachtet, wie man das eigentlich erwarten sollte, da ja der Sympathicus gefässverengernde Fasern führt und demnach eine Röthung und Erhöhung der Hauttemperatur auf der verletzten Seite hätte eintreten müssen. Jedenfalls hatten sich diese Erscheinungen schon wieder ausgeglichen, als der Fall in unsere Beobachtung trat, denn nach den Erfahrungen von Braun stellen sich die normalen Verhältnisse in diesem Punkte nach kurzer Zeit, spätestens nach zwei Tagen wieder her. Ophthalmologisch nachweisbare Veränderungen, wie Erweiterung der Retinalgefässe, bestanden in unserem Falle nicht. Ebenso keine Störungen der secretorischen Thätigkeit, welche wohl ebenfalls nach ganz kurzer wieder verschwinden. Puls und Athmung zeigten gar keine Störung. Das ist leicht erklärlich, denn diese Fasern befinden sich viel tiefer. Erst kürzlich hat Wallenberg' ') gelegentlich einer Stichverletzung. im Rücken Störungen des Pulses und der Athmung beobachtet, welche nach kurzer Zeit wieder zurückgingen. Die Schlucklähmung ist meiner Ansicht nach als eine directe Verletzung der Weichtheile des Pharynx aufzufassen. Zumal da die kleineren Suggillationen auf eine Schädigung der Schlundmusculatur hinweisen. Vergleichen wir also den in unserem Falle sich bietenden Symptomencomplex mit den Beobachtungen von Bra un, der gewissermaassen Experimente am lebenden Menschen angestellt hat, so haben wir ein sich vollständig deckendes Bild. Hier wie dort Pupillenverengerung, sowie geringe Ptosis auf der verletzten Seite, dagegen keine Veränderung. der Spannung des Bulbus, keinen Enophthalmus, keine Secretionsanomalien, keine Veränderung von Puls und Athmung. Das einzig Differirende ist, dass wir in unserem Falle vasomotorische Störungen nicht beobachtet haben, doch dürfte sich das daraus erklären, dass der Patient erst 14 Stunden nach der Verletzung in unsere Behandlung$\mathrm{kam}$, in welcher Zeit diese Störungen schon vorüber sein konnten. Wir stehen also dem Gesagten zufolge nicht an, in unserem Falle die Diagnose auf Durchschneidung des Halstheils des Nervus sympathicus zu stellen.

1) Neurologisches Centralblatt. 1901. Nr. 19. 